\title{
Well-differentiated thyroid cancer: Thyroidectomy or lobectomy?
}

\author{
Wouter P. Kluijfhout MSc, Lorne E. Rotstein MD, Jesse D. Pasternak MD
}

1 healthy 43-year-old woman was referred to the surgeon with an incidental nodule on the right side of her thyroid, which had been found when she underwent magnetic resonance imaging (MRI) for mild neurologic symptoms. There was no family history of thyroid cancer, and her neck had not been exposed previously to radiation. Upon examination, a palpable mass that was firm and nontender was found within the upper pole of the right lobe of the patient's thyroid. There was no palpable adenopathy, and fibreoptic laryngoscopy was normal. The patient had normal serum levels of thyroid-stimulating hormone (TSH), triiodothyronine and thyroxine; therefore, a thyroid uptake scan was not indicated. Ultrasonography of her neck showed one solid, isoechoic nodule $(2.0 \mathrm{~cm})$, with several microcalcifications and regular borders that was located superiorly in the right lobe (Figure 1). There were no signs of extrathyroidal extension and no suspicious lymph nodes in the central or lateral neck. Fineneedle aspiration biopsy of the nodule showed a lesion classified as BETHESEDA category V under The Bethesda System for Reporting Thyroid Cytology, suspicious for thyroid cancer.

Based on these findings, the surgeon discussed two options with the patient: total thyroidectomy or lobectomy of the right thyroid. This patient qualified for thyroid lobectomy based on the new 2015 American Thyroid Association guideline for managing thyroid nodules and differentiated thyroid cancer in adults. ${ }^{1}$

The patient wanted to preserve her thyroid function and opted for lobectomy. Intraoperative examination of the neck showed no suspicious lymph nodes and no signs of extrathyroidal tumour growth. Postoperatively, the patient had pain that was controlled with acetaminophen, and she was discharged home after after a six-hour observation period.
Pathologic examination found a follicular variant of papillary carcinoma $(1.8 \mathrm{~cm})$ with negative margins and no vascular invasion. In addition, a clinically insignificant papillary microcarcinoma $(0.2 \mathrm{~cm})$ was found in the same lobe. Two lymph nodes found in the specimen were free of tumour. A diagnosis of a pT1bNOM0 (stage 1) welldifferentiated thyroid cancer was made.

At one-month follow-up, the patient's serum TSH level was 1.95 (normal 0.5-5) mIU/L, and no thyroid suppression treatment was necessary. The patient will have yearly follow-up examinations that include ultrasonography and blood tests (including TSH, thyroglobulin and antithyroglobulin antibodies).

\section{Discussion}

Thyroid cancer is the most common endocrine malignant disease and its incidence is rising, likely secondary to increased detection. ${ }^{2}$ In South Korea, where there is institutional screening, thyroid cancer is now the most common malignant disease. ${ }^{1}$

Most malignant tumours of the thyroid are well-differentiated thyroid cancer and are considered low risk. ${ }^{1}$ These are papillary and follicular carcinomas of the thyroid and have an excellent prognosis with a 20-year survival rate of 90\%-95\%. Less common malignant thyroid

\section{KEY POINTS}

- The revised American Thyroid Association guideline now considers thyroid lobectomy a viable alternative for patients with low-risk, welldifferentiated thyroid cancers of up to $4 \mathrm{~cm}$.

- Thyroid lobectomy has advantages for patients, including preservation of thyroid function and a lower risk of complications.

- Patients considering thyroid lobectomy should be made aware of the possibility of a second operation; the decision regarding the initial extent of surgery should balance risks and benefits.
Competing interests: None declared.

This article has been peer reviewed.

The authors have obtained patient consent.

Correspondence to: Jesse Pasternak, Jesse.Pasternak@uhn.ca

CMAJ 2016. DOI:10.1503 /cmaj.160336 
nodules have a poor prognosis: these comprise medullary and anaplastic subtypes, which will not be discussed in this article. Historically, all patients with well-differentiated thyroid cancer (nodules $>1 \mathrm{~cm}$ in size) have been advised to undergo total thyroidectomy. ${ }^{3}$ This advice was based mainly on data from 52173 patients with papillary thyroid cancer who were included in the National Cancer Database in the United States. ${ }^{4}$ This study reported that patients who had undergone total thyroidectomy had a better survival rate compared with those who had undergone lobectomy. 4

There is increasing evidence, however, that suggests that a substantial percentage of patients with thyroid cancer are being overtreated. A recent re-evaluation of data from the National Comprehensive Cancer Database that included 61775 patients with thyroid cancer found no benefit of total thyroidectomy over lobectomy for selected low-risk patients. ${ }^{5}$ This resulted in the most notable change in the 2015 guideline: in the absence of certain high-risk features (e.g., extrathyroidal extension, aggressive histology, macroscopic lymphadenopathy), patients with well-differentiated thyroid cancer of up to $4 \mathrm{~cm}$ are now eligible for thyroid lobectomy. ${ }^{1}$

\section{Therapeutic dilemma}

Previously, total thyroidectomy was recommended for all patients with well-differentiated thyroid

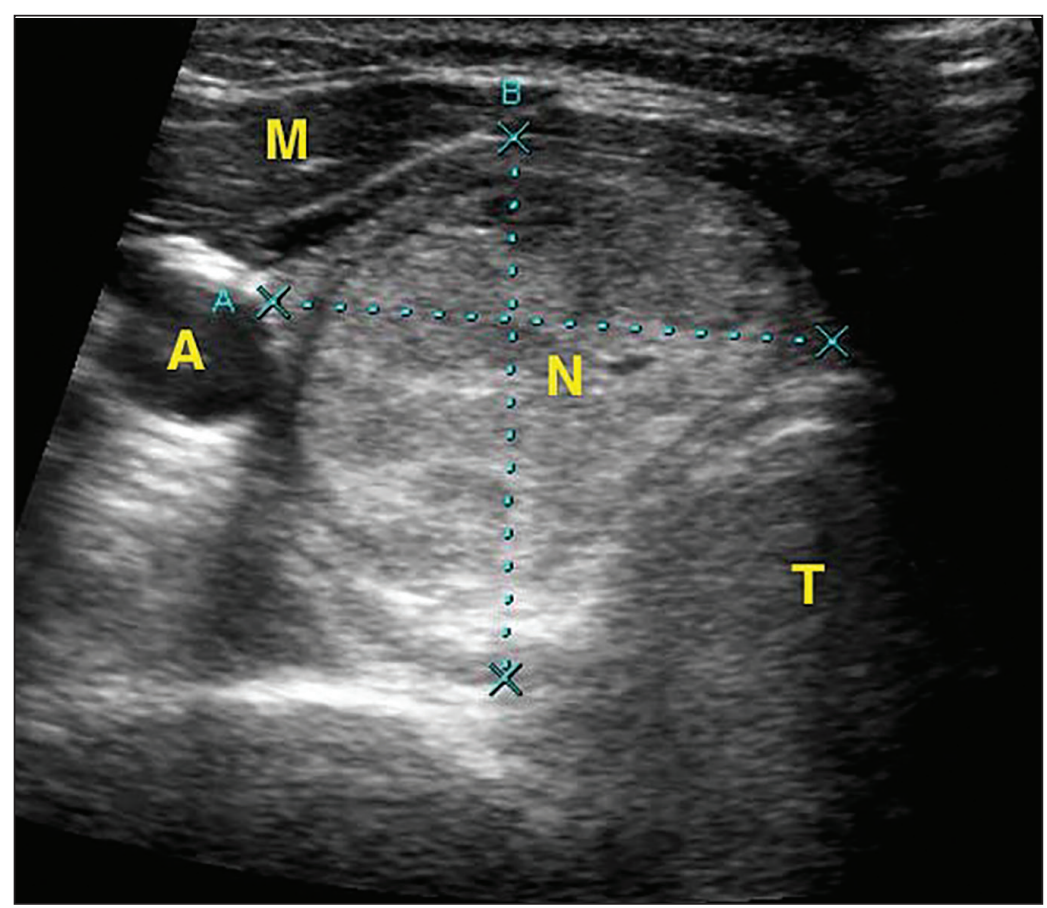

Figure 1: Transversal ultrasonography image of the neck of a 43-year-old woman showing a $2-\mathrm{cm}$ isoechoic nodule $(\mathrm{N})$ in the right lobe of the thyroid. Surrounding structures are the strap muscles of the neck (M), carotid artery (A) and trachea $(T)$. Light blue $A$ and $B$ refer to instrument distance measurement. cancer larger than $1 \mathrm{~cm}$ and decisions about extent of surgery were straightforward. In addition, many of these patients received postoperative radioactive iodine ablation.

With the change to the guideline, a less extensive lobectomy is a viable alternative and has a lower overall risk of complications. Furthermore, the indications for treatment with radioactive iodine ablation have narrowed considerably. ${ }^{1}$

A main drawback to thyroid lobectomy in the treatment of low-risk thyroid cancer is the definition of low risk. Although the guideline uses well-described definitions to classify risk scores for patients, some high-risk characteristics only become apparent upon final pathologic examination of the surgical specimen.

This scenario is important for physicians who care for these patients to understand, because seemingly low-risk tumours can have high-risk characteristics after histopathologic analysis. Features that require further treatment include microscopic extrathyroidal extension, vascular invasion, aggressive histology and high-risk metastases of the lymph node (Figure 1). Patients in these situations need to undergo a second operation (completion total thyroidectomy), often to enable adjuvant radioactive iodine ablation to lower their risk of recurrence. Recent evidence shows that, in some centres, $30 \%-40 \%$ of patients may be upstaged to a higher risk category after undergoing thyroid lobectomy for thyroid cancer. ${ }^{6}$ Mitigating the risk of completion thyroidectomy likely rests with the experience and preoperative workup of a high-volume, multidisciplinary thyroid cancer team (Figure 2).

\section{Lobectomy versus total thyroidectomy}

\section{Total thyroidectomy}

Traditionally, the arguments in favour of total thyroidectomy centred on complete clearance and ablation of all thyroid tissue. Removal of the thyroid gland makes it easier to detect recurrences in the neck using ultrasonography and also enables the use of thyroglobulin as a tumour marker for interval follow-up. Technological advances in both high-resolution ultrasonography and sensitive thyroglobulin assays now allow early detection of recurrences with one thyroid lobe in situ, putting previously held dogma into doubt.

Another argument for total thyroidectomy is the ability to administer postoperative radioactive iodine ablation for remnant ablation and as adjuvant therapy. Small retrospective studies ${ }^{7}$ had suggested that this would improve outcomes; however, the necessity of adjuvant treatment with radioactive iodine ablation is currently being questioned. $^{7}$ A recent meta-analysis comparing 
total thyroidectomy in conjunction with radioactive iodine ablation to lobectomy found no benefit for staging, follow-up or prevention of recurrence in patients at low risk. ${ }^{8}$ In addition, the administration of radioactive iodine ablation can lead to severe inflammation of the salivary glands and has been associated with secondary malignant tumours. ${ }^{9}$ However, occurrence of these adverse effects is dose-related, and these effects will become less common when the current trend of using lower doses becomes widespread.

\section{Lobectomy}

Lobectomy as a surgical strategy for low-risk thyroid cancer has multiple advantages. Thyroid surgery has two main described complications that are minimized with lobectomy. First, although uncommon, an injury to the recurrent laryngeal nerve, which innervates the vocal cord, may lead to stridor and hoarseness. A bilateral injury, only possible with bilateral surgery such as total thyroidectomy, would require tracheostomy. Second, the risk of postoperative hypocalcemia is negated when using a unilateral neck exploration such as lobectomy, because only two of four parathyroid glands will be subject to trauma.

After lobectomy, thyroid hormone replacement is only necessary in as low as $10 \%$ of patients in some series and is dependent on preoperative TSH levels and the presence of microsomal antibodies. When hormone substitution is indicated, patients need a lower dose of levothyroxine and fewer adjustments to reach euthyroidism.

Recent findings from the North American Thyroid Cancer Survivorship Study showed that patients with thyroid cancer currently have a worse quality of life compared with patients with breast or colorectal cancer, even though their prognosis is much better. ${ }^{10}$ Thyroid testing and symptoms related to hypothyroidism/hyperthyroidism have the greatest impact on quality of life in these patients. Removing the need for complete thyroid hormone replacement may provide one of the greatest benefits of lobectomy.

Box 1 gives an oversight of the advantages of both surgical strategies.

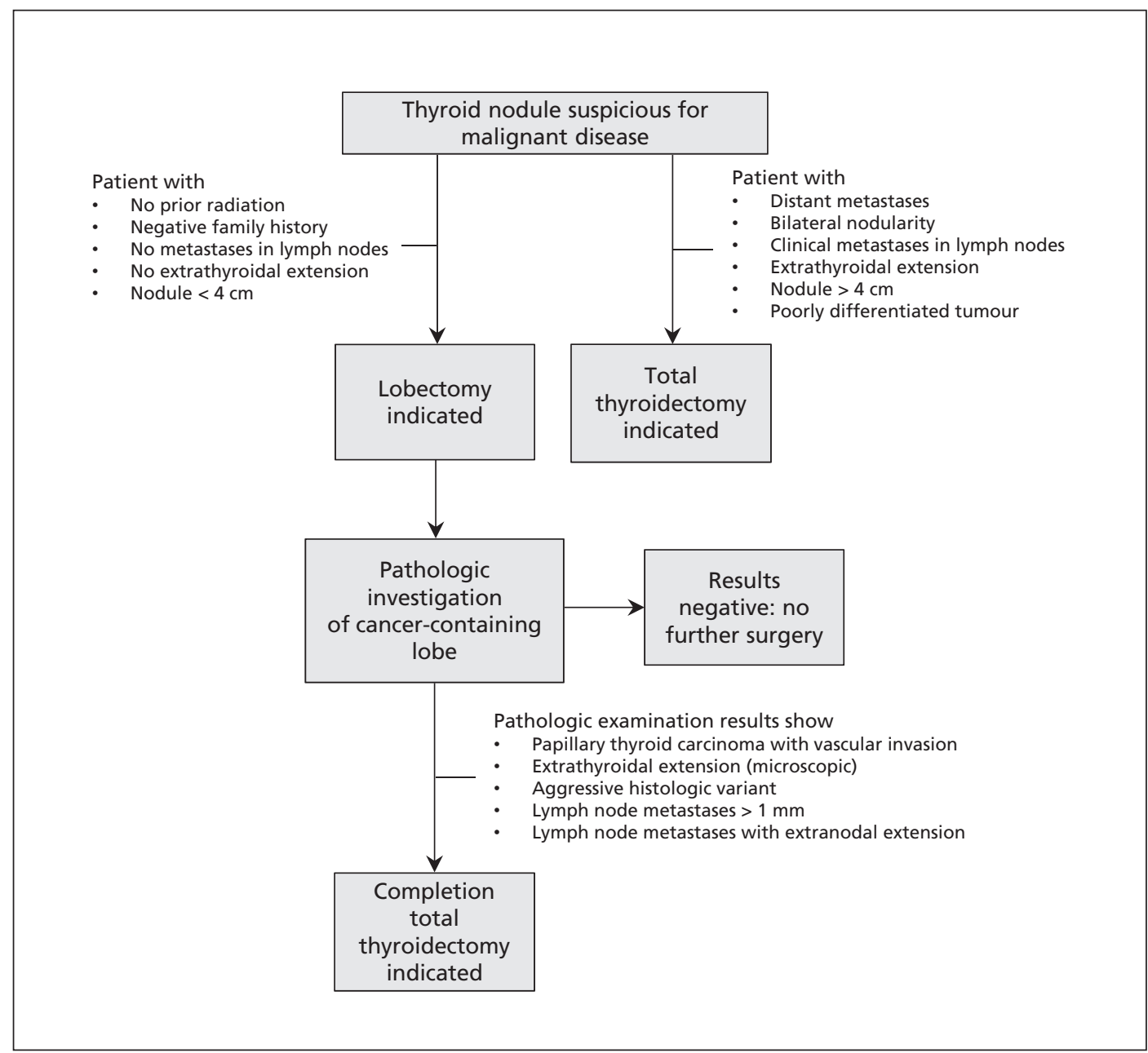

Figure 2: Eligibility of patients with thyroid cancer for lobectomy, and features that indicate need for completion total thyroidectomy. ${ }^{1}$ 
Box 1: Advantages of the two surgical strategies for thyroid cancer

Advantages of lobectomy

- Autonomous thyroid function most likely to be preserved

- No risk of hypoparathyroidism

- Less risk of recurrent laryngeal nerve palsy

Advantages of total thyroidectomy

- Ability to follow absolute thyroglobulin levels

- No need for a second operation to enable radioactive iodine ablation

\section{Conclusion}

The 2015 American Thyroid Association guideline recommends lobectomy for low-risk thyroid cancer of up to $4 \mathrm{~cm}$ in size. Therefore, patients with lowrisk thyroid cancer may avoid having thyroid hormone replacement and are not at risk of bilateral vocal cord paralysis or postoperative hypoparathyroidism. Although some patients will need a second operation based on their pathology report, the risks are low when the surgery is performed by an experienced high-volume endocrine surgeon. Both patients and physicians, who are ideally part of a multidisciplinary thyroid cancer team, should be aware of this recommendation when deciding the extent of surgery for low-risk thyroid cancer.

\section{References}

1. Haugen BR, Alexander EK, Bible KC, et al. 2015 American Thyroid Association Management Guidelines for Adult Patients with Thyroid Nodules and Differentiated Thyroid Cancer: The American Thyroid Association Guidelines Task Force on Thyroid Nodules and Differentiated Thyroid Cancer. Thyroid 2016;26:1-133.

2. Davies L, Welch HG. Increasing incidence of thyroid cancer in the United States, 1973-2002. JAMA 2006;295:2164-7.

3. Cooper DS, Doherty GM, Haugen BR, et al. Management guidelines for patients with thyroid nodules and differentiated thyroid cancer. Thyroid 2006;16:109-42.
4. Bilimoria KY, Bentrem DJ, Ko CY, et al. Extent of surgery affects survival for papillary thyroid cancer. Ann Surg 2007; 246:375-81, discussion 381-4.

5. Adam MA, Pura J, Gu L, et al. Extent of surgery for papillary thyroid cancer is not associated with survival: an analysis of 61,775 patients. Ann Surg 2014;260:601-5.

6. Kluijfhout WP, Pasternak JD, Lim J, et al. Frequency of highrisk characteristics requiring total thyroidectomy for $1-4 \mathrm{~cm}$ well-differentiated thyroid cancer. Thyroid 2016:26:820-4.

7. Mazzaferri EL. Thyroid remnant 131I ablation for papillary and follicular thyroid carcinoma. Thyroid 1997;7:265-71.

8. Lamartina L, Durante C, Filetti S, et al. Low-risk differentiated thyroid cancer and radioiodine remnant ablation: a systematic review of the literature. J Clin Endocrinol Metab 2015;100: 1748-61.

9. Sawka AM, Thabane L, Parlea L, et al. Second primary malignancy risk after radioactive iodine treatment for thyroid cancer: a systematic review and meta-analysis. Thyroid 2009; 19:451-7.

10. Aschebrook-Kilfoy B, James B, Nagar S, et al. Risk factors for decreased quality of life in thyroid cancer survivors: initial findings from the North American Thyroid Cancer Survivorship Study. Thyroid 2015;25:1313-21.

Affiliations: Division of General Surgery (Kluijfhout, Rotstein, Pasternak), University Health Network - Toronto, Toronto, Ont.; Department of General Surgery (Kluijfhout), University Medical Center Utrecht, Utrecht, Netherlands

Contributors: All of the authors made substantial contributions to the conception and design of the work and to drafting the manuscript and revising it critically for intellectual content, provided final approval of the version to be published and agreed to act as guarantors of the work.

The section Cases presents brief case reports that convey clear, practical lessons. Preference is given to common presentations of important rare conditions, and important unusual presentations of common problems. Articles start with a case presentation (500 words maximum), and a discussion of the underlying condition follows (1000 words maximum). Visual elements (e.g., tables of the differential diagnosis, clinical features or diagnostic approach) are encouraged. Consent from patients for publication of their story is a necessity. See information for authors at www.cmaj.ca. 